\title{
A Fast Large-Signal Model for Coupled-Cavity TWT's
}

\author{
VISHNU SRIVASTAVA AND RICHARD G. CARTER
}

\begin{abstract}
A fast and accurate one-dimensional large-signal model for coupled-cavity TWT's has been developed that can be used interactively for the optimization of a TWT design. Different modeling techniques for enhancing the speed of the large-signal model with no loss in accuracy are discussed in this paper. It has been shown that the speed of the model can be increased by 1) suitable selection of the basic integration parameters such as the number of electron discs per RF cycle and number of integration steps per cavity, 2) choosing a suitable method of integration of the relativistic equation of motion, 3) optimizing the iteration processes through each cavity and each section, and 4) efficient calculation of the space-charge forces. The model has been tested for the low-space-charge high-efficiency NASA CTS 200-W tube and for a high-space-charge high-gain tube. The characteristics of a 58-cavity tube for a single value of input power and frequency can be computed in less than $1.5 \mathrm{~min}$ of CPU time on a VAX 11/785 computer. This is at least 5 to 6 times faster than the previous models with the same accuracy.
\end{abstract}

\section{INTRODUCTION}

$\mathrm{C}$ OUPLED-CAVITY traveling-wave tubes [1] are high-power broad-band microwave amplifiers. These devices are widely used in space communications and in modern radar systems because of their unique characteristics of high gain, wide bandwidth, and high average power handling capability. They have many other favorable characteristics such as linear phase response and low noise. In the operation of these devices, the amplification of the RF wave is produced by the interaction between an electron beam and the electromagnetic wave propagating along a bandpass coupled-cavity slow-wave structure. The coupled-cavity structure is severed into sections in order to achieve higher gain without regenerative instabilities, and the phase velocity of the circuit is reduced near the output (velocity taper) in order to obtain higher electronic efficiency.

A theoretical analysis of the large-signal behavior of a coupled-cavity TWT is of immense interest because a tube is normally operated near to the maximum available output power. However, the computer simulation of a coupled-cavity TWT to analyze the electron-wave interaction

\footnotetext{
Manuscript received March 18, 1988; revised July 26, 1988. This work was supported in part by the English Electric Valve Company, Ltd. V. Srivastava was supported under a scholarship from the Association of Commonwealth Universities.

V. Srivastava was on leave with the Department of Engineering, University of Lancaster, Lancaster LA1 4YR, United Kingdom. He is with the Central Electronics Engineering Research Institute, Pilani, India.

R. G. Carter is with the Department of Engineering, University of Lancaster, Lancaster, LA1 4YR, United Kingdom.

IEEE Log Number 8823888.
}

process is very complicated at large-signal level. Therefore, computer models have been developed with a number of simplifying assumptions to make them fast enough to run interactively while retaining the desired accuracy and flexibility. Vaughan [2] developed a one-dimensional large-signal model of a coupled-cavity TWT with particular emphasis on increasing the speed of the program. Connolly and O'Malley [3] and Sung [4] improved Vaughan's model for better accuracy. These models take typically 10 to $20 \mathrm{~min}$ of CPU time for converged output for the NASA CTS $200-W$ tube. Therefore, it was desirable to further improve the speed of the large-signal program.

\section{The Large-Signal Analysis}

The large-signal analysis has been made for single-frequency operation of a coupled-cavity TWT (monochromatic operation) and for an unmodulated beam at the tube entrance. The analysis has been done on a cavity-by-cavity basis and under the fundamental assumption of onedimensional electron motion. For the analysis, the entering beam in one RF cycle is divided into a number of equal cylindrical charge discs (Fig. 1) and each charge disc is tracked in the laboratory frame whose coordinate system has its origin at the beginning of the first cavity. Each cavity is divided into a number of equal parts of length $\Delta z$ as shown in Fig. 2. The shooting method [5] has been used for solving the large-signal equations. The method uses stepping-in-distance through each cavity; the equation of motion for each charge disc is integrated at each distance step in the presence of the RF circuit field and space-charge field forces. The fundamental component of the RF beam current is calculated at each step of integration by Fourier analyzing the distribution of the electrons over one RF cycle. The RF beam current at each step is then used to calculate the forward- and backwardinduced voltage components in a cavity by integrating individual induced current components over the cavity period using Simpson's rule [5].

Since the resultant RF circuit field in a cavity depends on the electron trajectories and vice versa, this calculation is iterated through the cavity until a converged solution is obtained. This process is repeated for each cavity of a section in turn, and then the process is iterated through the section to account for the backward wave. The above analysis is repeated for each section of a tube until the last section, which finally gives the tube performance at a sin-

0018-9383/88/1100-2068\$01.00 (C) 1988 IEEE 


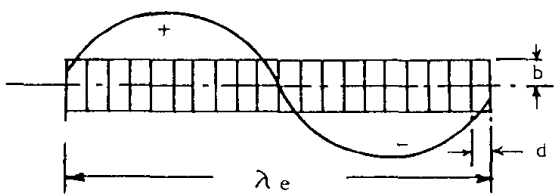

Fig. 1. Representation of the electron beam in one RF cycle by disc mode ( $b$ is the beam radius, $d$ is the disc thickness, and $\lambda_{t}$ is the beam wavelength).

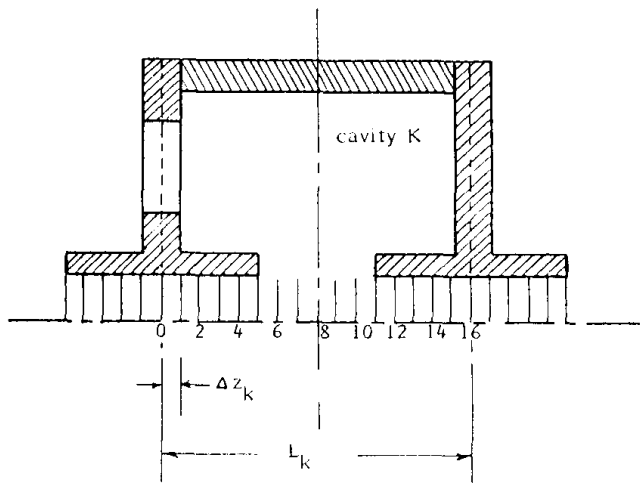

Fig. 2. Division of a cavity period into small steps for forward integration within a cavity ( $L$ is the cavity period divided into small steps $\Delta z$ in cavity $K$ ).

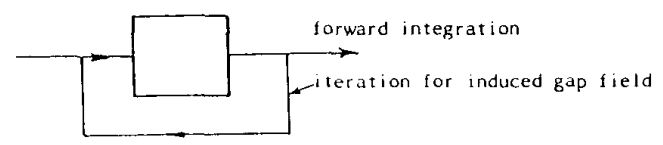

(a)

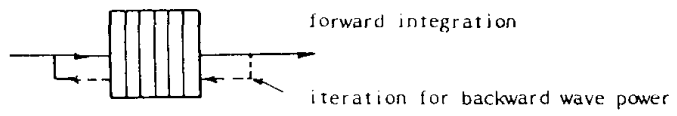

(b)

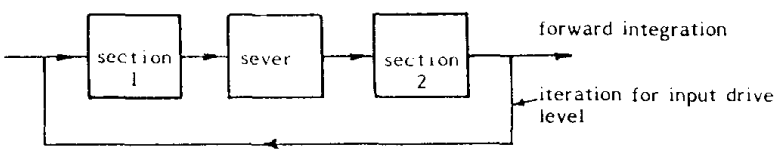

(c)

Fig. 3. Basic iteration mechanism during the forward integration process (a) Single cavity (b) Circuit section (more than one cavity). (c) Tube (combination of circuit sections and severs).

gle value of frequency and drive power. The whole process is iterated through the tube to analyze the tube performance for different cases. Fig. 3 illustrates the basic mechanism of the forward integration process as used by Kino et al. [6]. In the analysis, the working equations are primarily the same as given by Connolly and O'Malley [3] except that some of these equations have been corrected (see the Appendix).

\section{Modeling Techniques for a Fast Computer MOdEL.}

Different modeling techniques have been investigated to increase the speed of the one-dimensional large-signal program as much as possible without loss of accuracy. The structure of the program and programming techniques were also optimized to increase the program efficiency [7]. The modeling techniques are discussed below using as illustrations the results obtained from the program for the low-space-charge high-efficiency NASA CTS 200-W tube and for a high-space-charge (beam perveance of $1.22 \mu \mathrm{p}$ ) high-gain EEV tube.

\section{A. Integration Parameters}

In the program, provisions have been made for the choice of the basic integration parameters, i.e. the number of electron discs per beam wavelength and the number of integration steps per cavity. This is very useful for increasing the speed and accuracy of the program.

1) Number of Discs per RF Cycle: In general, the accuracy of the solution increases with the number of discs per RF cycle $\left(N_{d}\right)$, but so does the required computation time and the storage space on a computer. Because the space-charge force on every representative electron must be calculated as a function of the relative position of every other electron, the computation time increases rapidly with the increase of the number of discs per RF cycle. The provision for selecting different numbers of electron discs per RF cycle along the tube is based on the following results.

Fig. 4(a) shows the CPU time and the output efficiency versus number of discs per RF cycle used for computation of the high-space-charge tube. The drive power is chosen near to the saturation drive level of the tube. Fig. 4(b) shows profiles of the Fourier fundamental component of the RF beam current $\left(I_{1} / I_{0}\right)$ along the tube for different number of discs per RF cycle. Both figures show the selfconsistency of the tube output after 20 discs per RF cycle, but the CPU time is increasing almost parabolically. The self-consistency of the model was also checked for drive power above the saturation drive. It has been concluded from the results of different cases that, for calculation up to and including saturation, 24 discs per RF cycle are sufficient. Beyond saturation, more discs ( 36 or 48 ) should be used especially for high-efficiency tubes where debunching of the beam is high at the output.

It is obvious from Fig. 4(a) and (b) that an efficient numerical algorithm is possible if the number of discs per RF cycle is chosen to be small in the linear region and increased subsequently near to the saturation region. The program has been modified to choose 12 discs per RF cycle up to a cavity in the linear region where the number of discs per RF cycle is increased to 24 by an interpolation method. The interpolation of the trajectory data from 12 to 24 discs must satisfy the conditions that the kinetic energy of the beam and the fundamental RF beam current are conserved. Since in the linear region the electron trajectories are almost linear, the following techniques are 


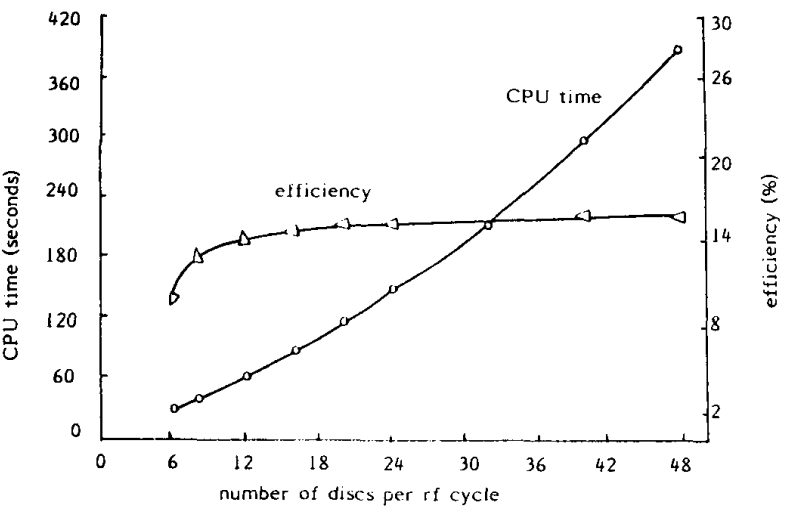

(a)

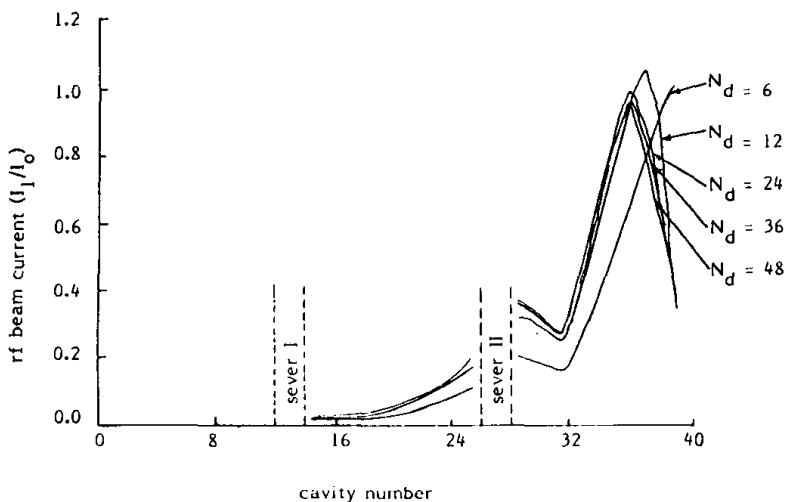

(b)

Fig. 4. (a) CPU time and output efficiency versus number of electron discs per RF cycle for the EEV tube: number of cavities (with severs) is 40 , number of integration steps per cavity is 16 , and number of iterations through a section is 5. (b) Profiles of the fundamental RF beam current along the EEV tube for different number of discs per RF cycle.

used for interpolation of the velocities and times of the discs.

First, the velocities and times of discs 1 to 12 are defined to be those of discs $1,3,5, \ldots, 23$. The velocity and time of disc 25 are determined from the trajectory data of disc 1 using the periodicity in time. Then the velocities and times of discs $2,4,6, \ldots$, and 24 are respectively defined from the following equations:

$$
\begin{aligned}
& u_{j}=\sqrt{\frac{u_{j-1}^{2}+u_{j+1}^{2}}{2}} \\
& t_{j}=\frac{t_{j-1}+t_{j+1}}{2} .
\end{aligned}
$$

This approach can easily be adapted so that different numbers of discs per RF cycle can be used for each section of a device, enabling the CPU time to be reduced appreciably while the accuracy remains high.

2) Number of Steps per Cavity: In the numerical integration, there is a range for the number of the integration steps per cavity $\left(N_{z}\right)$ that can be used for a given accuracy of the solution. Within this range, it is desirable to choose the smallest number of computational steps per cavity for the maximum speed. The lower limit on the number of the integration steps per cavity is imposed by the truncation error as well as by the instability of the computed solution. When the number of the integration steps per cavity is less than some minimum value, the computed signal levels oscillate with increasing amplitude around the correct functional values. The upper limit on the number of the integration steps per cavity is determined by the onset of effects from the round-off error and the increase in the CPU time. In the model, a provision has been made for selecting different step lengths in each cavity based on the following results.

Fig. 5(a) shows the CPU time and the output efficiency versus number of steps per cavity for the CTS tube for drive power near to the saturation drive. Fig. 5(b) shows the profiles of the fundamental RF beam current $\left(I_{1} / I_{0}\right)$ for different step lengths. Both figures show that the output is unstable if the number of steps per cavity is equal to 4 , and it is self-consistent if the number of steps per cavity is equal to or greater than 16 . It has been found that the self-consistency of the program does not change with the number of steps per cavity even if the drive power is increased beyond the saturation drive. These results indicate that the speed of the model can be increased without loss in accuracy by selecting a smaller number of steps per cavity in the early cavities and a greater number of steps in the later cavities of a tube.

\section{B. Method of Integration}

1) The one-dimensional relativistic equation of motion for each particle is used for tracking of electrons. This equation is expressed in terms of distance as an independent variable [3] and it is integrated with respect to distance at each step within a cavity. Integration with respect to distance is preferred because of its advantages in speed and accuracy over integration with respect to time [2]. The main advantage of distance-step integration is that the trajectories, i.e., the velocities and arrival times of the representative electrons are directly known as a function of distance. This is useful for the instantaneous calculation of the RF circuit field, the RF beam current, the induced voltage on the circuit, and the kinetic energy of the modulated beam at each plane along the circuit. It is only necessary to track the electrons in one RF cycle because of the periodicity of the system in time that exists under steady-state conditions. The distance step method is also useful for the analysis in a section after a sever, where the RF forward voltage is set to zero and the variables (velocity and time) characterizing the motions of the electrons are continuous.

2) The integration of the equation of motion is carried out by Euler's method [5] and by the Predictor-Corrector (successive iteration) method [5]. It has been found in the successive iterative method that using more than one iteration does not improve the accuracy. Fig. 6 shows the efficiency profiles for the EEV tube, using both Euler's method and Predictor-Corrector method for integration. 


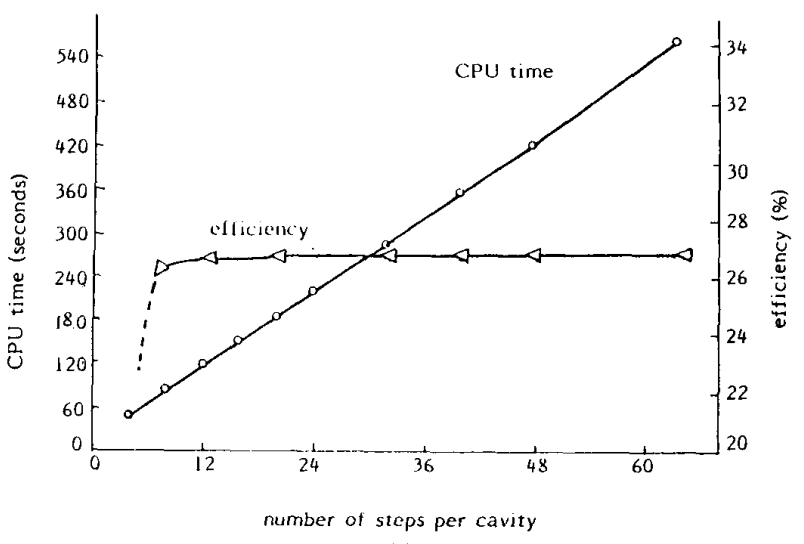

(a)

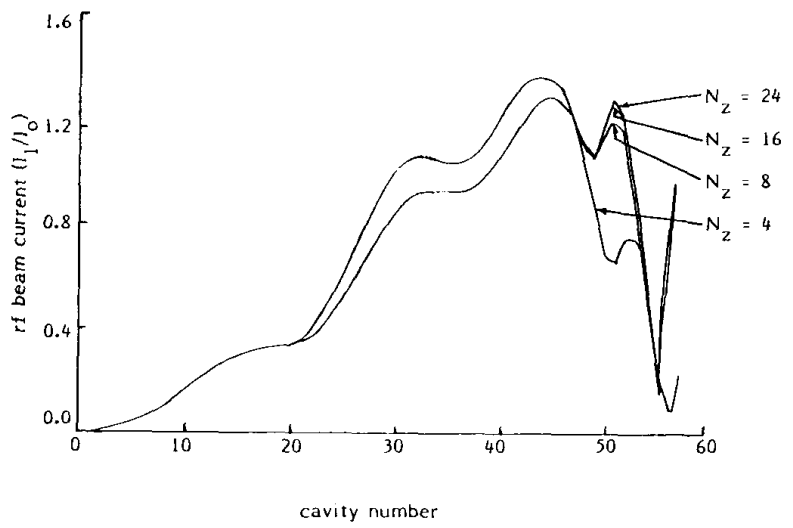

(b)

Fig. 5. CPU time and output efficiency versus number of integration steps per cavity for the CTS tube: number of cavities (with severs) is 58 , number of electron dises per RF cycle is 24 , and number of iterations through a section is 4. (b) Profiles of the fundamental RF beam current along the CTS tube for different number of integration steps per cavity.

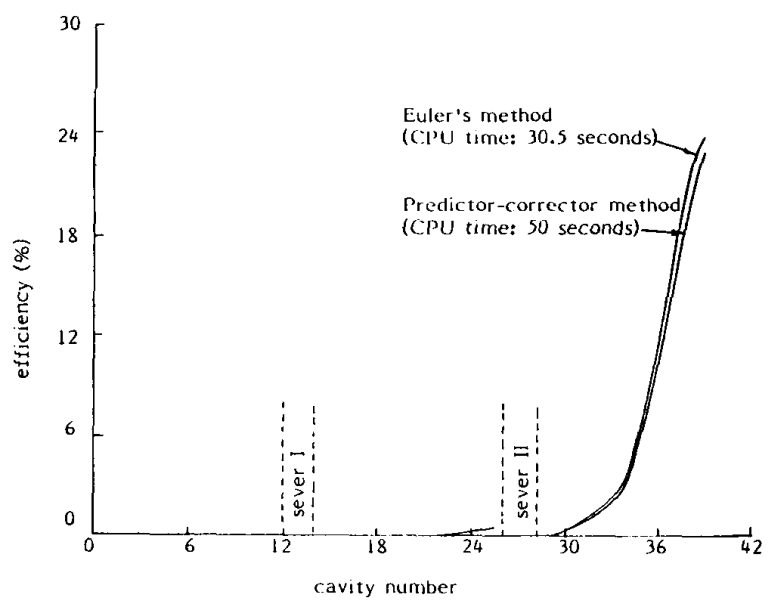

Fig. 6. Efficiency profiles along the EEV tube by Euler's and PreditorCorrector methods of integration: number of electron dises per RF cycle is 24 , number of integration steps per cavity is 16 , number of iterations through a cavity is 1 , and number of iterations through a section is 1 .

In the latter method, the results were computed with recalculation of the space-charge forces during the correction to the predicted values. The CPU times for both cases are also indicated. In the program, provision has also been made to use the same values of the space-charge force for correction as calculated during prediction (discussed in Section III-D).

In the program, the Predictor-Corrector method has been preferred over the Leap-Frog method (a noniterative method of integration) used by Connolly and O'Malley [3] for the following reasons: In the Leap-Frog method, two solutions are carried along: one with the values of $X$ computed at the alternate points $z_{1}, z_{3}, z_{5}, \ldots$ based on the values of $X^{\prime}$ at the intermediate points $z_{2}, z_{4}, z_{6}$, etc. The other solution is the values of $X$ computed at $z_{2}, z_{4}$, $z_{6}, \ldots$ based on the values of $X^{\prime}$ at $z_{1}, z_{3}, z_{5}$, etc. The Leap-Frog method, therefore, requires more variables and more core storage. It is also possible that the two solutions may diverge for a large value of $z$.

The storage of the trajectory data for a cavity has been optimized so that, when a new cavity is entered, the new trajectory data occupy the same storage space as the previous data. This has reduced the memory requirements and improved the program speed.

\section{Iteration Processes}

All the information required for calculating the RF field force on the electron beam by the circuit and for calculating the induced voltage components on the circuit by the modulated beam at each step of integration within a cavity is precalculated and stored. This approach has the advantage of making the program faster than the approach [3] of calculating these tables as each different cavity type is entered. In the program the iteration processes through each cavity and each section of a tube during the forward integration have been optimized as follows.

1) During the first pass through a section, the integration is performed only once for each cavity. On second and subsequent passes through the section, the program decides the number of passes for each cavity. This is achieved by determining the difference between the forward gap voltages in a cavity on this pass and on the previous pass through the section. If this difference is greater than 1 percent, then the program makes a second pass through the cavity. It has been found that in most cases, two passes through a cavity are sufficient for good accuracy. If the number of passes required for a cavity is more than one then the space-charge forces calculated in the previous pass through the cavity can sometimes be used in the subsequent passes through the cavity (discussed in Section III-D). This approach has the important effect of enhancing the speed with no loss in accuracy.

2) It has been found that when iteration is done on a section-by-section basis, it leads to a faster convergence in respect of the tube output results than iterating over the whole tube. The program stops the iteration through a section when a convergence of the order of 1 percent in the output power is achieved. In most cases, three to four passes through a section have been found sufficient except near the band edges where the effect of the backward wave becomes significant. Sometimes more passes ( 8 to 10 ) 


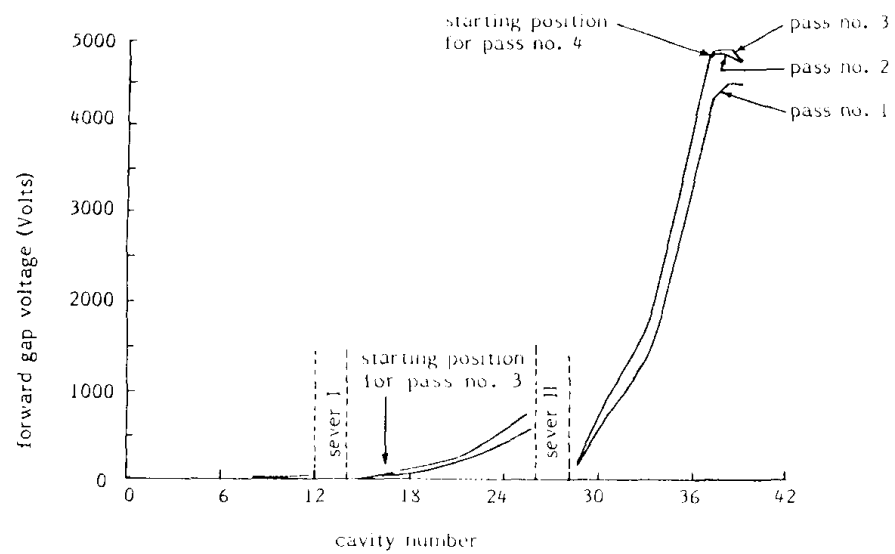

Fig. 7. Profiles of the forward gap voltage along the EEV lube in different passes through cach section of the tube

through a section are needed to achieve convergence at the output. In such a case, the modification given in 3 ) below helps to achieve faster convergence.

3) In programs described in the literature, each pass through a section was started from the first cavity of the section. It has been found during this investigation that the changes in the forward gap voltage during subsequent passes become significant only after some cavities (Fig. 7) especially in the input sections of a tube. This suggests that the next pass through the section should be started from the cavity (as marked in the figure) at which the difference in the forward gap voltage of this pass and the previous pass through the section is greater than a predefined value.

The program decides the starting position on a section during second and subsequent passes through the section. These passes are started from the cavity at which the difference between the forward gap voltages on this pass and on the previous pass through the section is greater than 1 percent. This modification has been found important for the first two sections of a tube as it helps to achieve a converged solution in the sections quickly after the second pass.

4) It has been found that, using a higher speed at the cost of less accuracy in the first pass through a section affects, neither the accuracy of the converged output results nor the rate of convergence, i.e., the number of passes required for output convergence, is not increased. Therefore, the program has been modified to make only one pass through a cavity and to do the forward integration at alternate steps in each cavity during the first pass through a section. If the number of steps per cavity is 16 , then the program does the integration at steps $0,2,4,6$, $8, \ldots, 16$ in the first pass through the section. This allows the same number of steps per cavity to be chosen for all the passes.

\section{Calculation of the Space-Charge Forces}

In a large-signal model, the approach to the calculation of the space-charge forces is a very important factor as it can save a lot of computing time if the calculation is done efficiently. The space-charge field force is calculated by setting up a look-up table; this table contains the coefficients of the quadratic equations derived from the spacecharge forces between two discs for relative positions varying from 0 to half a beam wavelength in small steps $(\Delta d)$ equal to $\left(\lambda_{c} / 2 \cdot l_{s p}\right)$ : where $\lambda_{c}$ is the beam wavelength and $I_{s p}$ is the number of points in half a beam wavelength.

The coefficients at the $n$th point, $A(n), B(n)$, and $C(n)$, calculate the space-charge force $(s c f)$ between two discs whose separation is $(z=n \Delta d)$ from the equation

$$
\operatorname{scf}(z)=A(n) z^{2}+B(n) z+C(n) .
$$

Thus, the space-charge force can be calculated at any intermediate point using the coefficients of the look-up table. The calculation of the space-charge force by this method has been optimized by: 1) improving the method of determining the relative disc positions, 2) efficient use of the look-up table, 3) using a constant space-charge force on a disc over a certain number of integration steps in a cavity, and 4) using the space-charge forces from the previous pass.

1) The computation for calculating the relative positions of the discs has been programmed for optimum speed by avoiding all possible repeated calculations and assuming that discs are moving with constant velocity during the minimum separation $(\Delta t)$ in time of arrival of the $i$ th and $j$ th discs at a plane $z$

$$
\begin{aligned}
& z_{i, j}=\Delta t \cdot u_{j} \\
& z_{j . i}=\Delta t \cdot u_{j} .
\end{aligned}
$$

In $4(\mathrm{a}), z_{i, j}$ is the relative position of the $j$ th disc from the $i$ th at a time $t_{i}(z) ; t_{i}(z)$ and $u_{j}(z)$ are the arrival time of the $i$ th and the velocity of the $j$ th discs at a plane $z$. respectively. It has been found that (4) is sufficiently accurate for calculating the relative disc positions, and it is not necessary to use a second-order term to improve the accuracy.

2) The number of data points in the look-up table containing the coefficients of the quadratic equations is a pro- 
TABLE I

Comparison Between Two Different Approaches of Using the LookUP TABLE

\begin{tabular}{l|c|c|c|c}
\hline \hline Data Points & Quadratic Incerpolation & \multicolumn{2}{|c}{ Direct Look-up } \\
\hline & Eff cr.(\%) & $\begin{array}{c}\text { CPU Time } \\
\text { (sec.) }\end{array}$ & Effen.(\%) & $\begin{array}{c}\text { CPU Time } \\
\text { (sec.) }\end{array}$ \\
\hline 60 & 22.25 & 34.61 & 24.93 & 31.18 \\
\hline 120 & 22.12 & 34.59 & 25.16 & 31.22 \\
\hline 240 & 22.08 & 34.96 & 24.65 & 31.31 \\
\hline 480 & 22.06 & 35.29 & 23.71 & 31.54 \\
\hline 1200 & - & - & 22.82 & 31.98 \\
\hline
\end{tabular}

number of electron discs per $r$ cycle $=24$ number of integration steps per cavity $=16$ number of integrationstens per cavity $=16$ Mumber of Iteration through a cavity number of lceration through a section $=1$ and the space charge force was calculated at each step of integration.

gram input parameter and it was set to 240 . The results for a range of data points in the look-up table varying from 60 to 480 in the program are presented in Table I, for the high-space-charge EEV tube.

Table I also compares the above approach with another method of using the look-up table. In the latter method, the look-up table contains the values of the space-charge force between two discs at relative positions varying from 0 to half a beam wavelength in small steps, and the spacecharge force at a distance was taken directly from the table for the point nearest to that distance. Table I shows that calculating the space-charge force from the quadratic interpolation is more accurate and the difference in the CPU time in both methods is not very great. In the program, a provision was also made to calculate the spacecharge force using linear interpolation. However, in the case of linear interpolation, more data points are required in the look-up table to obtain the same results as in the case of quadratic interpolation. Therefore, in the computation, the coefficients for quadratic interpolation were preferred so that the accuracy could be ensured even in a high-space-charge tube.

3) Since the space-charge force acting on a disc is the resultant force due to all the other surrounding discs, it does not change rapidly across each cavity during a pass through the section especially at the input end (Fig. 8). Therefore, the same value of the space-charge force can be used over a certain number of integration steps $\left(N_{\text {step }}\right)$.

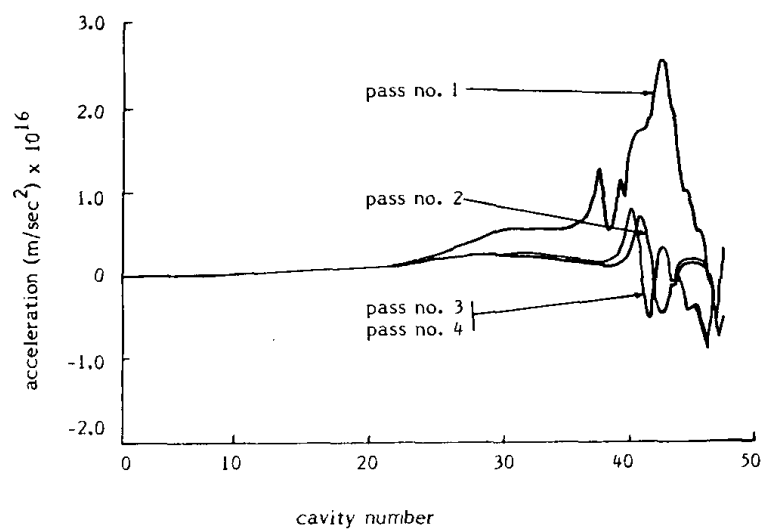

Fig. 8. Variation of the acceleration on a disc due to space-charge force along the CTS tube in different passes through the tube.

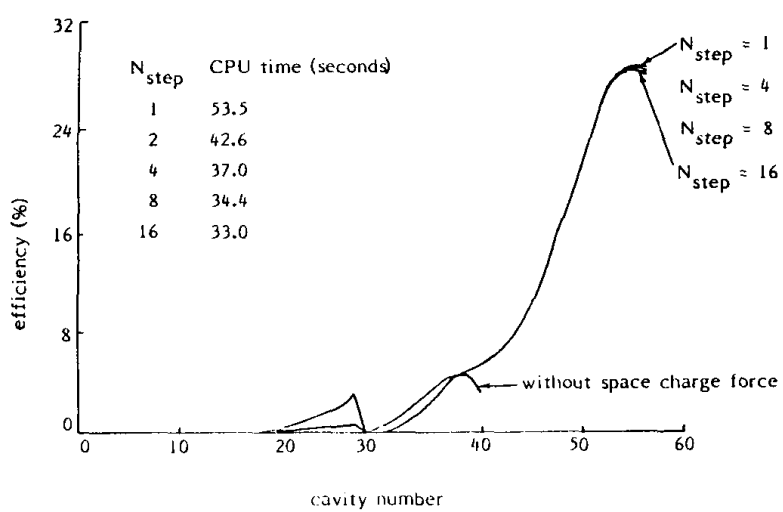

Fig. 9. Efficiency profiles along the CTS tube for varying the number of integration steps for a uniform space charge force on a disc: number of electron discs per RF cycle is 24 , number of integration steps per cavity is 16 , number of iterations through a cavity is 1 , and number of iterations through a section is 1 .

In the program, a provision has been made for calculating the space-charge force at $1,2,4,8$, and 16 steps. For a step interval equal to 1 , the space-charge forces are calculated at each step of integration within a cavity. At the other extreme, the space-charge forces are calculated only at the beginning of a cavity and are assumed constant throughout it. Fig. 9 shows the efficiency profiles for the CTS tube for different values of step interval in the calculation of the space-charge forces. The CPU time for each case is also indicated. It is interesting to note that the efficiency profile for step interval equal to 16 does not differ much with the efficiency profile for step interval equal to 1 . The figure also shows the efficiency profiles without the space-charge force, with illustrates that the results with and without the space-charge forces are very different. These results suggest that initially the spacecharge force on a disc can be calculated with a higher value of step interval and the step interval for the calculation of the space-charge force can be reduced successively if the error in the space-charge force exceeds some limit.

The program has been modified so that it can be run with a higher value of step interval in calculation of the 
space-charge forces and, in the forward integration process, the program decides at the beginning of each cavity the number of the integration steps for which the spacecharge force on a disc is kept constant. If the number of the integration steps with uniform space-charge force is greater than one, then this number is halved when the difference between the space-charge forces on a disc, calculated in the normal manner and by extrapolating the last three calculated values of the space-charge force on that disc, is greater than 1 percent.

4) Fig. 8 shows that the space-charge force on a disc at a point does not change much for successive passes after the first, especially in the linear region. Therefore, the same values of the space-charge forces can be used in these passes if the difference between the space-charge forces on a disc at a point on this and the previous passes is not more than 1 percent.

The program stores the space-charge forces calculated in the first pass through a section. In the next pass through the section the program decides at the beginning of each cavity, from the difference between the space-charge forces on a disc on this and the previous pass, whether they should be recalculated in the cavity. If recalculation of the space-charge forces is not desired, then the program uses the stored values of the space-charge forces on the discs in the cavity. This approach has approximately doubled the computational speed in a typical case with no loss in accuracy of the results. However, one limitation of this approach is that it requires a large memory in the computer to store the array of the space-charge forces on each disc at each integration point along the whole tube. The solution to this problem is to store the data at some positions, and to calculate the space-charge forces at the intermediate points by interpolation.

\section{Speed and Accuracy of the Model}

When the modifications described were incorporated in a one-dimensional large-signal model of a coupled-cavity TWT, the program was found to be faster than previous programs with no loss in accuracy. On a VAX 11/785 computer, the characteristics of the NASA CTS 200-W tube for a single value of input power and frequency can be computed in less than $1.5 \mathrm{~min}$ of CPU time, which is at least 5 to 6 times faster than previous models having the same accuracy. The increased speed of this program is a major advantage for interactive applications. The model is self-consistent, and it also provides a comprehensive range of output data for design purposes. The program generates both tabulated and graphical output; the principal graphical outputs are the Applegate diagram, velocity-phase diagram, profiles of the RF circuit field and RF beam current characteristics along the tube, and the spent beam characteristics.

The internal energy balance check was typically satisfied within -0.15 percent of the input beam power even beyond saturation showing the high numerical accuracy of the model. The error, which is maximum at the position of maximum beam bunching, appears because the

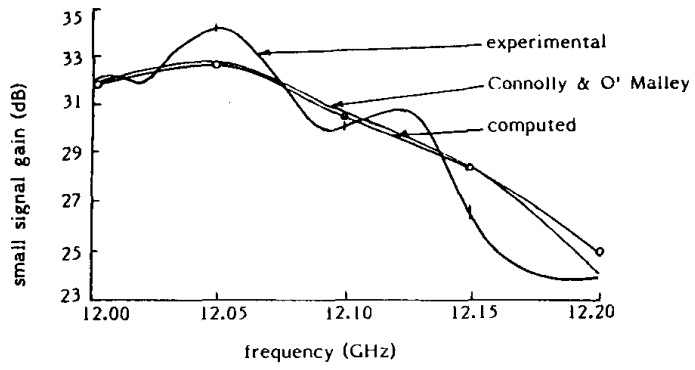

(a)

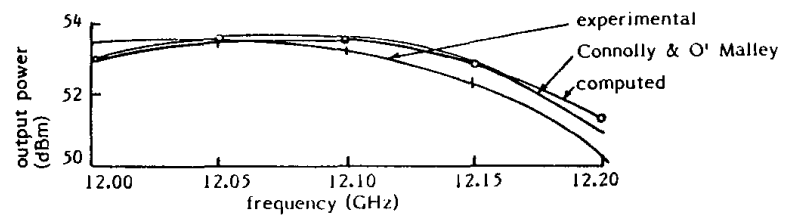

(b)

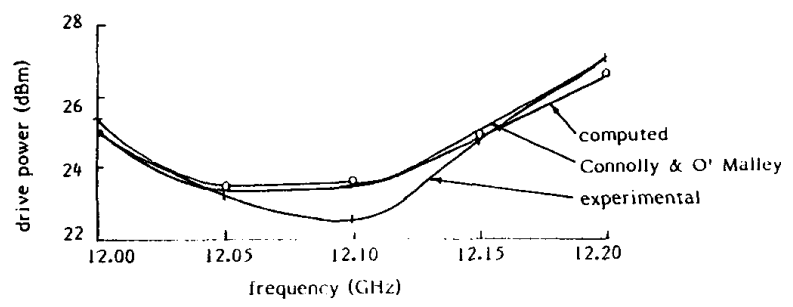

(c)

Fig. 10. Computed and experimental results for the NASA CTS 200-W tube on: (a) small-signal gain versus frequency (0-dBm drive), (b) saturated output power versus frequency, and (c) saturation drive power versus frequency.

change in the space-charge potential energy is neglected in the energy balance calculation.

The accuracy of the program was also checked by comparing the computed results with the experimental results for two typical space harmonic tubes. Fig. 10 shows a good agreement between the computed results on (a) the small-signal gain, (b) the saturated output power, and (c) the saturation drive power with the corresponding experimental results [3] over the frequency band for the lowspace-charge high-efficiency NASA CTS 200-W tube. The figure also shows that the agreement between the computed results and the results of Connolly and O'Malley [3] is excellent. Fig. 11 shows results on the gain versus frequency for the high-space-charge high-gain EEV tube. The figure shows good agreement between the computed results and the experimental results over the scaled operating band of 2.96 to $3.12 \mathrm{GHz}$. The poor agreement between the computed results and the experimental results outside the operating band might be due to errors in the values of the parameters used for the matching and the slot tapered cavities of the tube that were calculated from their dimensions using an equivalent circuit program [8]. 


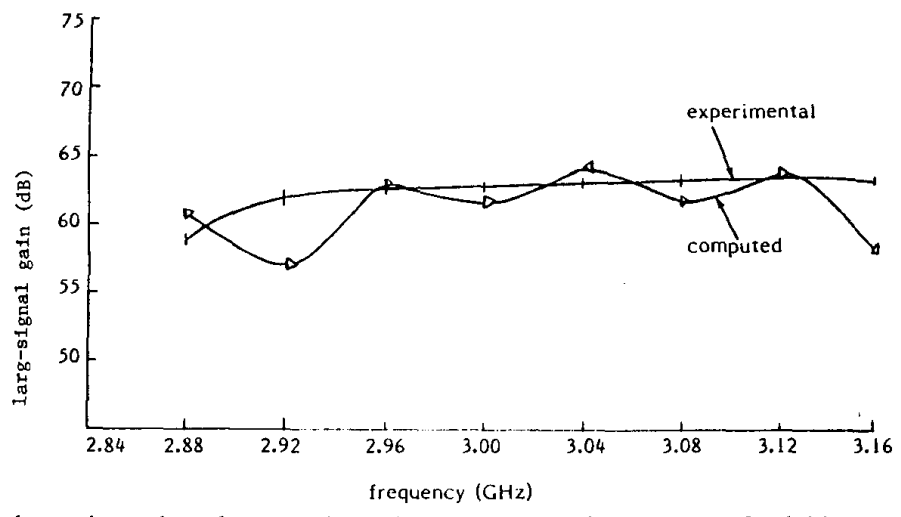

Fig. 11. Computed and experimental results on the large signal gain versus frequency at a fixed drive power for the EEV tube.

\section{Conclusions}

A one-dimensional large-signal model of a coupledcavity TWT has been developed that is much faster than other similar programs with the same accuracy. The basic theory of the model is based on the large particle method in which the electron beam is modeled in terms of the cylindrical charge discs and the axial movements of the charge discs are tracked in response to the RF circuit and space-charge field forces. Since the success of a computer model is judged by the extent to which it predicts and agrees with the experimental results, different modeling techniques were investigated that could increase the speed of the program with no loss in accuracy.

1) A provision has been made for selecting different numbers of representative electrons per beam wavelength along the tube, i.e., fewer electrons in the linear region and more in the nonlinear region. Though this approach is very useful for increasing the speed of the program, there is a need to improve it in the nonlinear region. Similarly, a provision has been made for selecting different step lengths in each cavity, i.e., a small number of steps in the early cavities and more in the later cavities.

2) The integration of the equation of motion has been carried out with respect to distance because of its advantages in speed and accuracy over integration with respect to time. The Predictor-Corrector method has been found better than the Leap-Frog method [3] because the storage space and the computation time required for the same accuracy are less.

3) The iterations through each cavity and through each section during the forward integration process have been optimized. It has been shown that iteration on a sectionby-section basis leads to a faster convergence and that the first pass through a section can be made faster at the cost of less accuracy. It has also been shown that, instead of making each pass from the first cavity of the section, a later cavity may be used as the starting position for the third and subsequent passes through the section.

4) Since efficient calculation of the space-charge force can save a lot of computing time, the program was optimized by: improving the method of determining the relative disc positions, efficient use of the look-up table, suitable choice of the number of integration steps for the uniform space-charge force on a disc, and using the space-charge force from the previous pass.

With these modifications, the program speed has been considerably improved to, typically, less than $1.5 \mathrm{~min}$ of CPU time for converged output on a VAX type 11/785 computer. Although the program has been checked only for a typical low-space-charge high-efficiency tube and for a typical high-space-charge high-gain tube, it is hoped that the program is generalized enough to be used for different types of tubes with good accuracy and speed. Also, the model can easily be modified to investigate the interaction of a traveling-wave circuit with a prebunched beam by assuming the current and velocity distributions at the entrance to the circuit. This approach can be used for studying different velocity taper circuits in the output section of a tube.

\section{APPENDIX}

In the large-signal analysis, the working equations primarily differ from that used by Connolly and O'Malley [3] in the following respects:

1) The effect of the space-charge potential depression under the actual boundary condition is taken into account for calculating the dc beam velocity [9]. Because of the radial variation of the potential depression, the dc beam velocity is no longer the same for electrons at different radii. It has been assumed that the beam velocity on the axis can be used with sufficient accuracy because some degree of compensation is produced by variation of the beam velocity about its axis due to magnetic focussing field.

2) The equations for calculating the RF circuit field acting on the electron beam and for calculating the voltage induced on the circuit by the modulated beam require summations over an infinite set of space harmonics. It has been found that summation from -2 to 2 is generally sufficient.

3) The equation for the RF circuit field force on an electron disc differs from [3, eq. (20)] by a negative sign that was found by its derivation.

4) The equation for the RF beam current density differs 
from [3, eq. (74)] by a factor of 2 in the numerator that was found from the theory of Fourier series of complex arguement [10].

5) The equation for the forward and backward components of the induced voltage differ from the respective $[3$, eqs. (76) and (77)] by multiplication factor 0.5 that were found by their derivatives.

6) In the power balance verification, equations related to the RF circuit power differ from the respective [3, eq. (55) and (56)] by a factor of half of the induced voltage with the forward or backward gap voltage. This additional term appeared in these equations on the basis of the power balance within the cavity.

\section{ACKNOWLEDGMENT}

We wish to thank the management of the English Electric Valve Co., Ltd. for permission to publish this paper. V. Srivastava wishes to thank the Association of Commonwealth Universities for awarding him a scholarship and the Director, CEERI, Pilani, India, for sponsorship to carry out research studies at the University of Lancaster. He is also thankful to Dr. S. S. S. Agarwala and S. $\mathrm{N}$. Joshi of his organization for their encouragement.

\section{REFERENCES}

[1] J. F. Gittins, Power Travelling Wave Tubes. London: English Universities Press, 1964

[2] J. R. M. Vaughan, "Calculations of coupled cavity TWT performance,"' IEEE Trans. Electron Devices, vol. ED-22, pp. 880-890, 1975

[3] D. J. Connolly and T. A. O'Malley, "Computer program for analysis of coupled-cavity traveling-wave tubes," NASA Rep. TND-8492, May 1977.

[4] W.-M. Sung, "Large signal theory and computation for coupled-cavity TWTs," Acta Electron. Sin., vol. 12, no. 3, pp. 25-32, 1984.

[5] R. W. Hamming, Numerical Methods for Scientists and Engineers. New York: McGraw-Hill, 1973.

[6] G. S. Kino, Y. Hiramatsu, W. A. Harman, and J. A. Ruetz, "Smallsignal and large-signal theories for the coupled-cavity TWT," in Proc. 6ih Int. Conf. Microwave and Optical Generation and Amplification, pp. 49-53, 1966.

[7] D. Van Tassel, Program Style, Design, Efficiency, Debugging, and Testing. Englewood Cliffs, NJ: Prentice-Hall, 1978.

[8] R. G. Carter and L. Shun-kang, "Method for calculating the properties of coupled cavity slow wave structures from their dimensions," IEE Proc., vol. 133, pt. H, no. 5, pp. 330-334, 1986
[9] V. Srivastava and R. G. Carter, "Effect of boundaries on the space charge potential in coupled-cavity travelling wave tubes, "IEE Proc. vol. 133 , pt. I, no. 5 , pp. 185-188, 1986.

[10] D. K. Cheng, Analysis of Linear Systems. Reading, MA: AddisonWesley, 1959

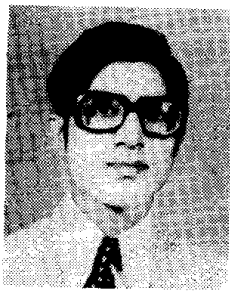

Vishnu Srivastava was born in Ajmer, India on November 22, 1953. He received the B.Sc. and M.Sc. degrees from the University of Rajasthan, Jaipur, India, in 1973 and 1975, respectively, and the Ph.D. degree in engineering from the University of Lancaster, Lancaster, United Kingdom, in 1987. His Ph.D. dissertation concerns the largesignal modeling of coupled-cavity TWT's.

Since 1976, he has been working in the Microwave Tubes Area, Central Electronics Engineering Research Institute, Pilani, India, where he has been engaged in the design and development of high-power microwave tubes. He was a Commonwealth Scholar at the University of Lancaster from October 1984 to September 1987. His current research interests are in CAD/CAM of high-power microwave tubes and UHV technology.

Dr. Srivastava is an Associate Member of the Institution of Electrical Engineers (U.K.) and a member of the Institution of Electronics and Telecommunication Engineers (India), the Computer Society of India, and the Indian Physics Association.

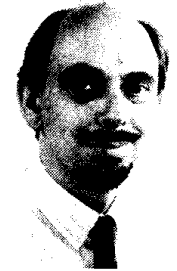

Richard G. Carter graduated in physics from Queens' College Cambridge in 1965 and received the Ph.D. degree from the University of Wales in 1968 for work on wave propagation on neutralized ion beams under the supervision of Dr. R. H. C. Newton.

From 1968 to 1972, he was a Development Engineer with the English Electric Valve Company Limited (EEV) working on high-power travelingwave tubes. In 1972, he became a Lecturer in the Department of Engineering of the University of Lancaster. He was responsible for the development of the B.Sc. course in physical electronic engineering and the M.Sc. course in project engineering. He is the author of a number of papers on microwave tubes and on engineering education, and of the undergraduate text Electromagnetism for Electronic Engineers. A Consultant to EEV since 1976, he was promoted to Senior Lecturer and EEV Fellow in 1986.

Dr. Carter is a Chartered Engineer and a Member of the Institution of Electrical Engineers (U.K.). 